\title{
A Function to Describe Attenuation of Cosmic Ray Air Shower Particles in Snow
}

\author{
The IceCube Collaboration ${ }^{1}$, \\ ${ }^{1}$ http://icecube.wisc.edu/collaboration/authors/icrc15_icecube \\ E-mail: krawlinseuaa.alaska.edu
}

\begin{abstract}
Snow overburden has become a part of the IceTop detector at the South Pole, and becoming more significant over time as snowdrift buries the array. Snow attenuates the electromagnetic component of cosmic ray air showers before they reach the detectors, reducing the measured signals, raising the threshold of the array in general, and introducing a potential source of systematic error in measuring shower energy. Understanding this attenuation is vital for shower reconstruction and energy resolution. A simulation of cosmic ray air shower particles has been used to map the attenuation response due to snow, and parameterize the behavior of the attenuation as a function of zenith angle and shower evolution stage.
\end{abstract}

Corresponding authors: K. Rawlins ${ }^{1 *}$

${ }^{1}$ University of Alaska Anchorage, Department of Physics and Astronomy

The 34th International Cosmic Ray Conference,

30 July- 6 August, 2015

The Hague, The Netherlands

\footnotetext{
${ }^{*}$ Speaker.
} 


\section{Overview: Why is a complicated snow reconstruction necessary?}

IceTop [1] is the surface component of the IceCube Observatory, composed of tanks of frozen water deployed at the surface of the Antarctic ice at the South Pole. Although the tanks were initially deployed with their tops flush with the snow surface, drifting snow at the site buries the tanks over time. The rate of accumulation is irregular over the array, but is about $20 \mathrm{~cm}$ per year on average. Figure 1 shows the snow coverage on the array, measured in three different years. Some tanks have very little snow overburden; others have close to 3 meters of coverage.
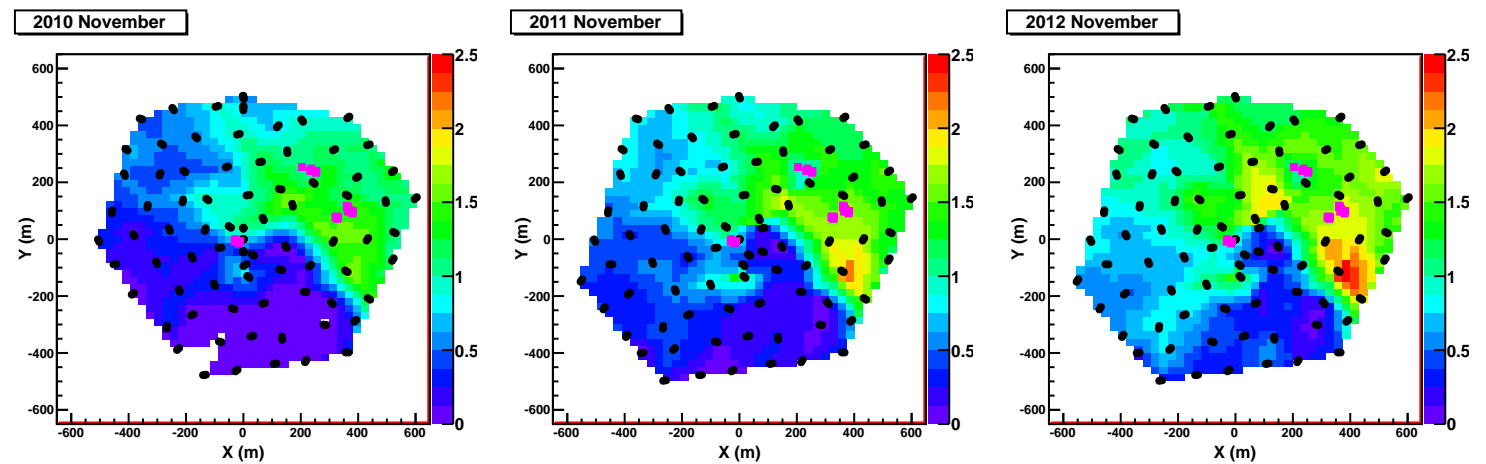

Figure 1: Snow accumulation over the IceTop detector, measured in three different years: 2010, 2011, and 2012. The pink dots indicate the positions of buildings at the site.

The charged particles in the air showers measured by IceTop get attenuated by any snow that is piled on top of a tank. A reconstruction algorithm must take into account these varying snow depths when computing the signals that are expected in a tank; one would expect smaller signals in a deeply-buried tank. But how much smaller?

In analyses published so far $[2,3,4,5]$ the expected signal in a snow-covered tank is attenuated by a simple exponential:

$$
S / S_{\text {nosnow }}=\exp \left(-x / \lambda_{\text {eff }}\right),
$$

where $x$ is the slant depth of the tank through the snow (equal to the snow depth $z$ divided by $\cos (\theta)$ ), and $\lambda_{\text {eff }}$ is the "effective attenuation length" due to snow. The same attenuation formula is applied uniformly to all tanks regardless of their distance from the core and in the same way for all showers regardless of their energy, composition, or zenith angle.

In reality, however, the physics of snow attenuation can be complicated in a variety of ways. For one thing, electrons and photons experience snow attenuation much more so than muons, and the relative contributions of electrons, photons, and muons to the tank signal depend on several factors, such as distance of the tank to the center of the shower and the shower morphology which depends on the energy, composition, and zenith angle of the shower. In addition, tank signals result not just from one incoming particle but a whole spectrum of them, and since the response of the snow depends on the particle energy, the shape of the spectrum affects the overall attenuation. The spectrum of the EM particles, like the fraction of signal from muons, depends on the distance to the core and the shower morphology. Where particle energies are very high (such as near the shower core), snow can even result in production of particles rather than attenuation [6]. When applying a "one size fits all" treatment to all tanks as we do currently, all of these effects are averaged over into 
a single "effective attenuation length". This effective $\lambda_{\text {eff }}$ cannot be predicted easily from theory, and varies from year to year as the tanks are buried by more snow and the signal becomes more dominated by muons [5].

A better way of correcting for snow is to: a) estimate the fraction of the signal which is electromagnetic, and apply a snow correction to only that fraction, and b) to develop a correction which is a function of the tank's distance to the core, and the shower's morphology. This work is a continuation of what was begun in [6], in which a simulation of IceTop-like tanks at discrete radii and under discrete snow depths is used to develop such a function. The expectation is that this more complicated function will replace the simple exponential, and result in better reconstructions of shower sizes and energies.

\section{The simulation}

Vertical (zenith $=0^{\circ}$ ) showers from proton and iron primaries were simulated using CORSIKA [7] and FLUKA [8], at discrete energies of $\log \left(E_{\text {primary }} / \mathrm{GeV}\right)=5.0,5.5,6.0,7.0$, and 8.0. A smaller sample of showers at non-vertical zeniths $\left(30^{\circ}, 45^{\circ}\right.$, and $\left.60^{\circ}\right)$ at these same energies were also produced. The showers were thrown at a fictitious ideal array comprised of 235 single IceToplike tanks arranged in rings of constant radius. The layout of the tanks is shown in Figure 2; there is a dense inner core of tanks at radii of 3,5, 7, and 10 meters, a series of tanks at intermediate distances (15 - 200 meters), and more sparsely-spaced rings out to 500 meters. The azimuths of the tanks in the dense inner core are offset so as to avoid physical overlap between the closely-spaced tanks.
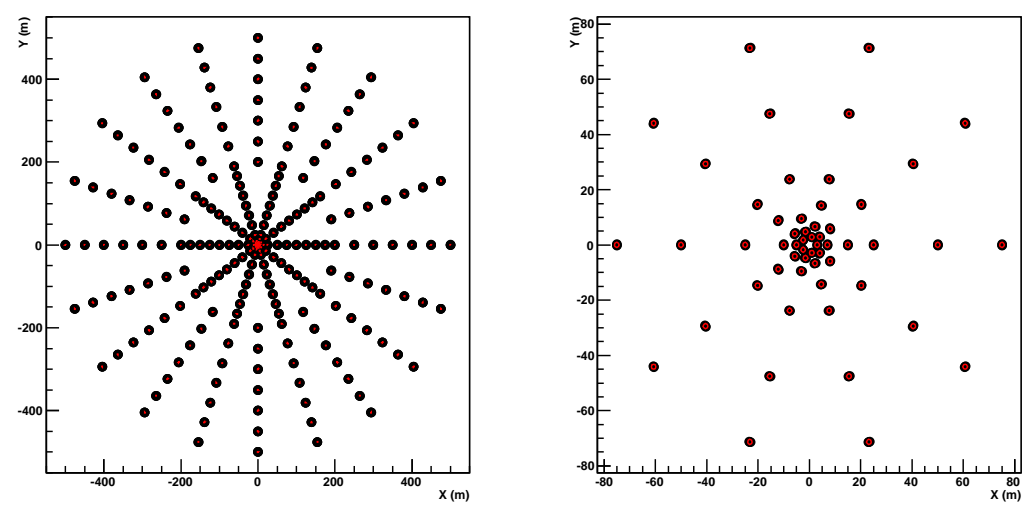

Figure 2: Layout of the tanks in the simulation. Left: the full array of tanks. Right: zoomed-in look at the tanks within 80 meters of the core.

A detector simulation package based on GEANT4 [9] is used to simulate the response of the tanks to the air shower particles. To restrict this study to electromagnetic particles only, all the muons in the shower are removed from the simulation before injection into the detector simulation. Photons which arrive at the photocathode of each DOM are recorded and counted. No simulation of other IceTop-specific downstream processes such as generating waveforms or triggers is performed; this study focuses on photoelectrons only. 
Each of the simulated primaries is aimed at the array's center $(x=0, y=0)$. However, because CORSIKA includes a magnetic field, the charged particles will experience some deflection. Since the shower as a whole carries a net positive charge, vertical events are deflected slightly to grid south of the origin in the CORSIKA coordinate system. When present, this small offset is taken into account when computing the distance to the shower core for each of the tanks in the simulation, and becomes significant only for the very close tanks at low energies.

Each set of showers is repeated with the same array under different uniform coverages of snow, with depths ranging from 0 to 4 meters.

\section{Attenuation curves}

For each primary energy and mass simulated, photoelectrons arriving at each tank are counted, and the results are sorted according to the radius

Table 1: Offsets in core location due to magnetic deflection (in meters along the y-axis).

\begin{tabular}{|l|l|l|}
\hline $\log (\mathrm{E} / \mathrm{GeV})$ & protons & iron \\
\hline 5.0 & -0.31 & -7.10 \\
\hline 5.5 & 0 & -2.44 \\
\hline 6.0 & 0 & -0.62 \\
\hline 7.0 & 0 & 0 \\
\hline 8.0 & 0 & 0 \\
\hline
\end{tabular}
of that tank from the shower core and the snow coverage over the tank. The results can be visualized with a set of "attenuation curves", such as those shown in Figure 3. Each small panel in this figure represents a different radius $r$ from the shower core. Within each panel is an attenuation curve $\left(S / S_{\text {nosnow }}\right.$ vs. snow depth $\left.x\right)$ for tanks at that radius. The entire figure depicts attenuation curves for a particular primary energy and mass (for instance "protons at $1 \mathrm{PeV}$ "); similar figures can be made for all primary energies and both masses. All of the curves, by construction, intercept the y-axis at a value of 1 .
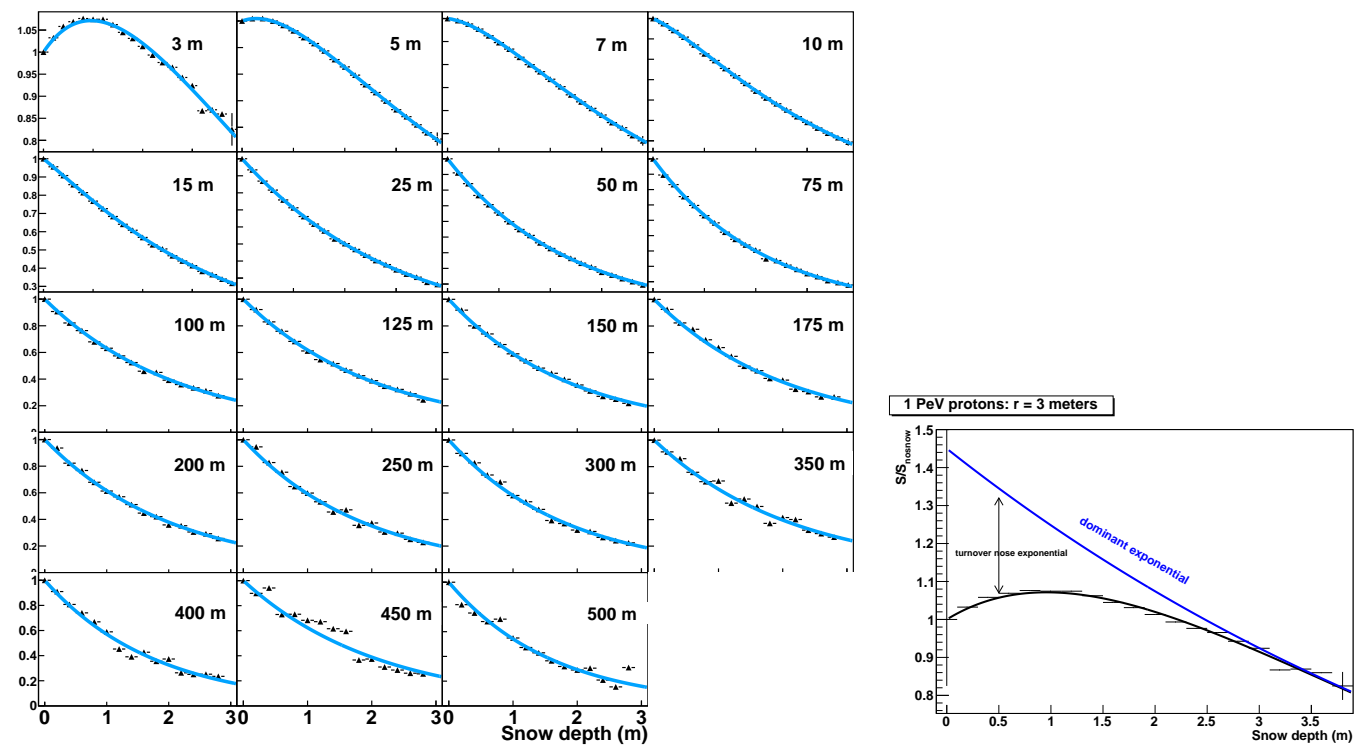

Figure 3: Left: Series of attenuation curves, for 16 different distances from the core (ranging from 3 meters to 500 meters), for $1 \mathrm{PeV}$ vertical proton primaries. Right: Sketch of the idea of a dominant exponential an an turnover nose term. Note: the blue curve is not an actual fit to this curve, but is drawn in by hand to visualize the construction of the general function. 
Beyond $r=30$ meters, all of the attenuation curves look like a simple exponential decay. But closer than $r=30$ meters, the attenuation curves exhibit signs of particle production at small depths, followed by eventual exponential attenuation deeper into the snow. A general form which fits all the attenuation curves well is a "dominant exponential with turnover nose":

$$
S / S_{\text {nosnow }}=c_{0} \exp (x \cdot s)-\left(c_{0}-1\right) \exp \left(x \cdot s_{\text {nose }}\right)
$$

The first term describes the "dominant exponential", which is evident in all tanks at all distances. Its slope is $s$, which one expects to be a negative number, and $c_{0}$ describes where the dominant exponential intercepts the y-axis. The second term describes the "turnover nose", which appears only for small radii, and is non-zero only if $c_{0}$ is greater than one. It is an upside-down exponential whose slope is $s_{\text {nose }}$, another number expected to be negative. Figure 3 (right) demonstrates the contributions of these two terms to the overall curve. For tanks further away than 30 meters, $c_{0}=1$, and the second term disappears.

First, all attenuation curves are fit to the function above, with $c_{0}$ restricted to 1.0 for tanks beyond 30 meters and all three parameters $\left(s, c_{0}\right.$, and $\left.s_{\text {nose }}\right)$ floating free for tanks within 30 meters. Next, we examine each of these parameters as a function of $r$, and primary energy/composition, to search for parametrizable patterns. It has been argued by $[10,11]$ that the measurable properties of an air shower are related to the number of particles at shower maximum, and the stage of shower evolution $t$ :

$$
t=\frac{X-X_{\max }}{X_{0}}
$$

where $X$ is the slant depth through the atmosphere traveled by the shower, $X_{\max }$ is the slant depth of shower maximum, and $X_{0}$ is the radiation length $\left(36.7 \mathrm{~g} / \mathrm{cm}^{2}\right)$. Therefore in the following sections we study the behavior attenuation curve parameters $\left(s, c_{0}\right.$, and $\left.s_{n o s e}\right)$ as a function of $t$, rather than as a function of primary energy and composition.

\section{1 $\mathbf{R}>30$ meters: the dominant exponential}

Figure 4 shows the fit values of $s$ for different showers as a function of radius. For the points beyond 30 meters radius, no turnover nose is allowed and $s$ is the only free parameter. Although the protons (solid symbols) and iron (open symbols) occupy very different regions of this plot, a composition-independent pattern arises when the points in Figure 4 are organized by average stage of shower evolution $t$, represented by colors. All the $s$ 's past 30 meters can be fit to a function of $t$ :

$$
s=d+e \cdot t+\gamma \log _{10}(r)
$$

where $d, e$. and $\gamma$ have best-fit numerical values shown in Table 2 .

\section{$3.2 \mathrm{R}<30$ meters: the turnover nose}

Once the fit to $s$ past 30 meters is complete, these numbers are held fixed, and the attenuation curve fits are repeated but with only $c_{0}$ and $s_{\text {nose }}$ allowed to float free. Once again, patterns related to $t$ emerge, as shown in Figure 5. As with $s, c_{0}$ and $s_{\text {nose }}$ can be fit to a general function of $t$ :

$$
c_{0}=A \exp \left(-r /\left(r_{0}+B \cdot t\right)\right)+1.0
$$




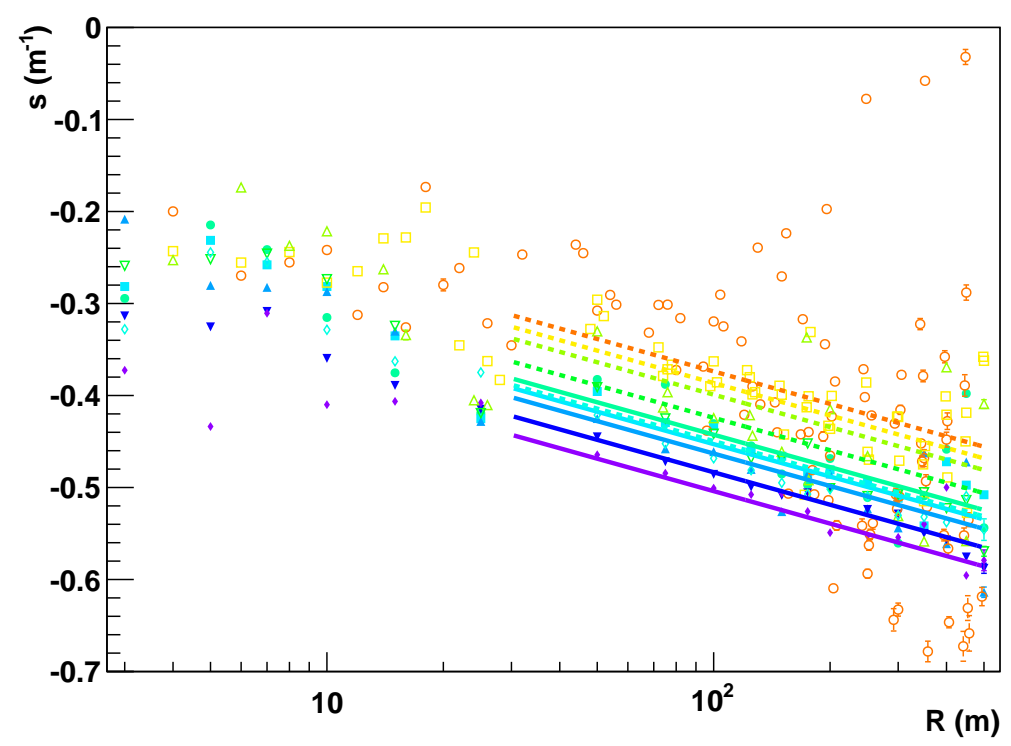

Figure 4: Fitting the exponential slope, using radii $>30$ meters. Solid points = proton showers, open points $=$ iron showers. The color of the points represents the stage of shower evolution $t$ (violet $=-1$, red $=10)$.
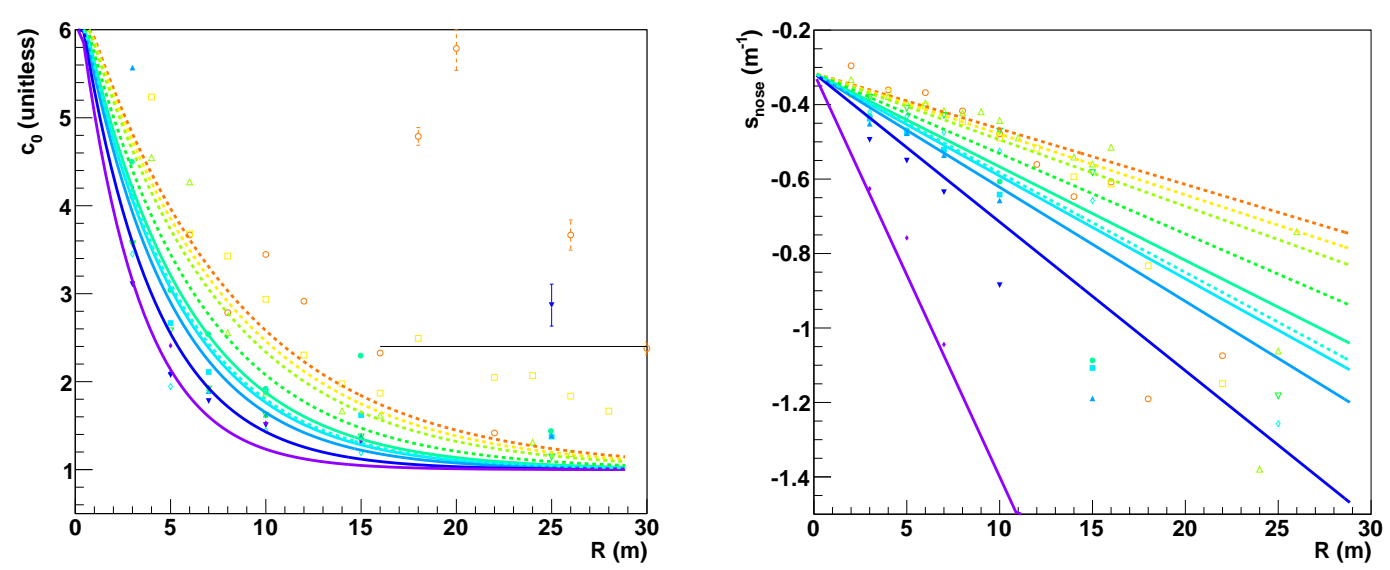

Figure 5: Fitting the features of the "turnover nose" $\left(\right.$ left $=c_{0}$, right $\left.=s_{\text {nose }}\right)$ at small radii. Solid points $=$ proton showers, open points $=$ iron showers. Stage of shower evolution $t$ is represented by color (violet $=-1$, red $=10)$.

$$
s_{\text {nose }}=a+\left(b \log _{10}(t+1.0)+c\right) r
$$

where $A, r_{0}, B, a, b$, and $c$ and additional best-fit numerical values shown in Table 2. As expected, $c_{0}$ approaches a value of 1 as the radius goes beyond 30 meters, and although $s_{\text {nose }}$ appears to diverge in this region, its effect is modulated by $c_{0}$ going to 1 , making it less and less relevant to the overall curve as 30 meters is approached. 
Table 2: Best-fit values for the parameters that go into the complete snow attenuation function. Values obtained when fitting only proton or iron showers are shown for comparison.

\begin{tabular}{|r|r|r|}
\hline parameter & both compositions & $\mathrm{p} / \mathrm{Fe}$ individually \\
\hline$d$ & -0.259 & $-0.263 /-0.293$ \\
\hline$e$ & 0.0133 & $0.0136 / 0.0195$ \\
\hline$\gamma$ & -0.116 & $-0.112 /-0.121$ \\
\hline$A$ & 5.57 & $6.88 / 5.80$ \\
\hline$B$ & 0.489 & $0.248 / 0.117$ \\
\hline$r_{0}$ & 3.63 & $3.40 /-0.756$ \\
\hline$a$ & -0.317 & $-0.321 /-0.334$ \\
\hline$b$ & 0.0312 & $0.0264 / 0.0232$ \\
\hline$c$ & -0.0457 & $-0.0486 /-0.0331$ \\
\hline
\end{tabular}

\section{Next steps: using this function in reconstruction}

In order to apply this function to actual IceTop cosmic ray air shower data, there are two additional functions which must be explored and quantified.

- Estimate the fraction of the signal which is electromagnetic. Near the core of a shower the signals in tanks should be dominated by EM particles, whereas at the edge of the shower it should be dominated by muons. The fraction of the signal which is electromagnetic $f_{E M}=$ $S_{E M} /\left(S_{E M}+S_{\mu}\right)$ is a function of the distance from the core $r$, and expected to vary from 1 at small $r$ to zero at large $r$. This function is expected to depend on the shower's energy, composition, and zenith angle.

To estimate this, IceTop detector simulations were run in a mode in which only certain particle components were propagated to the tanks (for instance, "electromagnetic only" or "muons only"), and the individual signals measured. The signals from these individual components as a function of radius can be fit to parabolas in log-log space $\left(\log _{10}(S)\right.$ vs $\left.\log _{10}(r)\right)$. The ratio of the electromagnetic curve to the total is shown in Figure 6. Fits to the log-log parabolas for the 0-degree and 25-degree curves (which are very similar in Figure 6) as a function of $\log E$ can be used to estimate $f_{E M}$ for each tank in real IceTop events according to their radius from the core and the reconstructed energy of the event.

\section{- Estimate the shower evolution t for each shower.}

Because IceTop only samples particles at ground level, estimating the stage of shower evolution with this detector is a challenge. In theory, the shower age should be related to $t$. The lateral distribution function model used for IceTop signals does include a slope parameter called $\beta$, which is related to the shower age [1], but this variable appears to not be robust for this purpose in initial testing. If the signal can be modeled by two lateral distribution functions, a Nishimura-Kamata-Greisen function for the electromagnetic component and a separate function for muons $[12,13]$, then the age of the electromagnetic component may be a better estimator. 

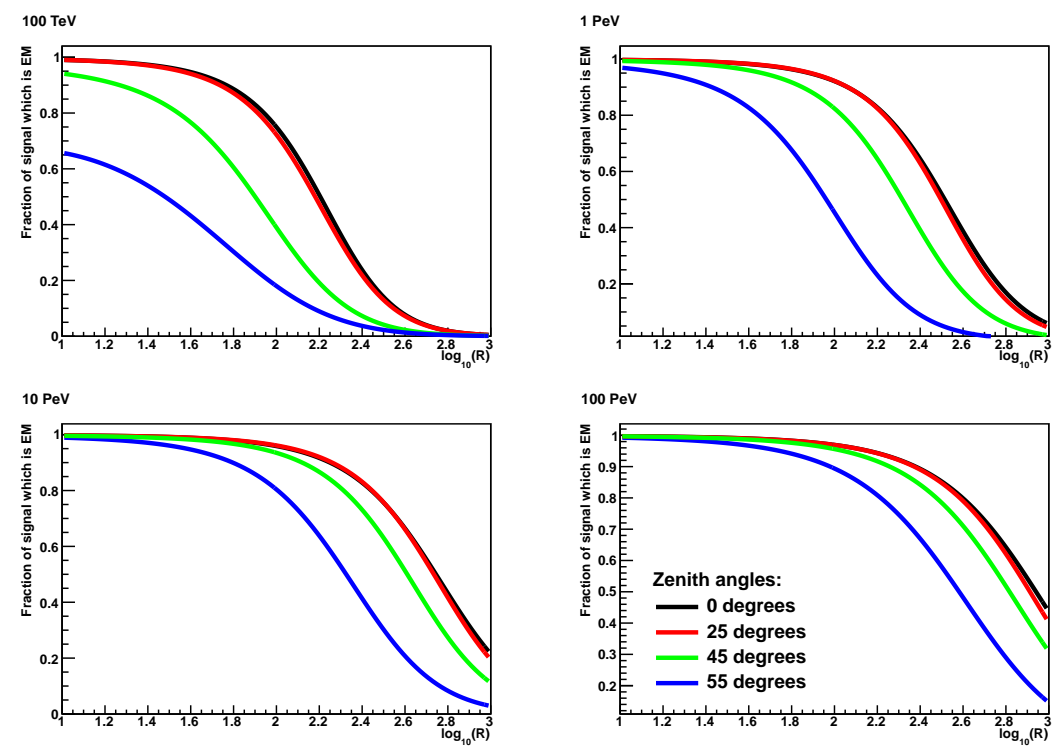

Figure 6: Fraction of the signal which is electromaganetic, broken down by energy (the four different panels) and by zenith angle (the four colors).

\section{References}

[1] R. Abbasi et al. NIM, A700, (2013) 188-220.

[2] R. Abbasi et al. (IceCube Collab.) Astropart. Phys., 42, (2013) 33.

[3] M.G. Aartsen et al. (IceCube Collab.) Phys. Rev. D, 88, (2013) 042004.

[4] T. Feusels et al. (IceCube Collab.) "Cosmic Ray Composition and Energy Spectrum between 2.5 PeV and $1 \mathrm{EeV}$ with IceTop and IceCube”, Proc. of the 33rd ICRC (Rio de Janiero), paper 0861 (2013)

[5] K. Rawlins et al. (IceCube Collab.), PoS(ICRC2015)334, these proceedings

[6] K. Rawlins et al. (IceCube Collab.) "The Effect of Snow Accumulation on Signals in IceTop", Proc. of the 33rd ICRC (Rio de Janiero), paper 1106 (2013)

[7] D. Heck et al., Report FZKA 6019 (1998).

[8] G. Battistoni et al., AIP Conference proceedings 896, (2007) 31.

[9] S. Agostinelli et al., Nucl. Instrum. Meth. A 506, (2003) 250.

[10] P. Lipari, Phys. Rev. D 79, (2009) 063001.

[11] S. Lafebre, et al. Astropart. Phys. 31, (2009) 243.

[12] J. Nishimura and K. Kamata, Progr. Theor. Phys. 6 (1958) 93.

K. Greisen, Ann. Rev. Nucl. Part. Sci. 10 (1960) 63.

[13] J. Gonzalez et al. (IceCube Collab.), PoS(ICRC2015)338, these proceedings 